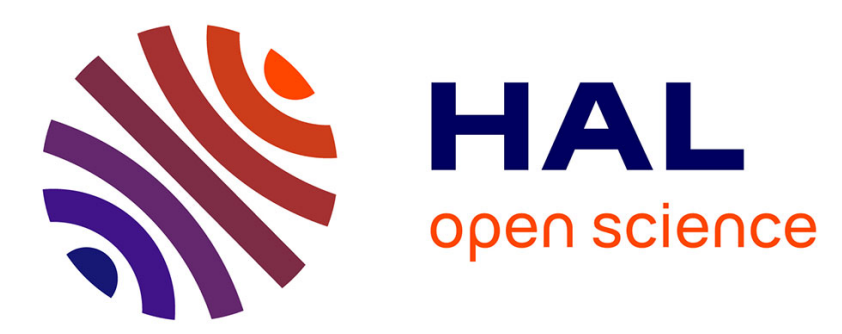

\title{
Astrometric detection of a low-mass companion orbiting the star AB Doradus
}

\author{
J. C. Guirado, J.E. Reynolds, Jean-Francois Lestrade, R. A. Preston, David L.
} Jauncey, D. L. Jones, A. K. Tzioumis, R.H. Ferris, E.A. King, J.E.J. Lovell, et al.

\section{To cite this version:}

J. C. Guirado, J.E. Reynolds, Jean-Francois Lestrade, R. A. Preston, David L. Jauncey, et al.. Astrometric detection of a low-mass companion orbiting the star AB Doradus. The Astrophysical Journal, 1997, 490 (2), pp.835-839. 10.1086/304920 . hal-02054083

\section{HAL Id: hal-02054083 https://hal.science/hal-02054083}

Submitted on 6 Mar 2019

HAL is a multi-disciplinary open access archive for the deposit and dissemination of scientific research documents, whether they are published or not. The documents may come from teaching and research institutions in France or abroad, or from public or private research centers.
L'archive ouverte pluridisciplinaire HAL, est destinée au dépôt et à la diffusion de documents scientifiques de niveau recherche, publiés ou non, émanant des établissements d'enseignement et de recherche français ou étrangers, des laboratoires publics ou privés. 
The AstrophysiCAL Journal, 490:835-839, 1997 December 1 (C) 1997. The American Astronomical Society. All rights reserved. Printed in U.S.A.

\title{
ASTROMETRIC DETECTION OF A LOW-MASS COMPANION ORBITING THE STAR AB DORADUS
}

\author{
J. C. Guirado, ${ }^{1,2}$ J. E. Reynolds, ${ }^{3}$ J.-F. Lestrade, ${ }^{4}$ R. A. Preston, ${ }^{1}$ D. L. Jauncey, ${ }^{3}$ D. L. Jones, ${ }^{1}$ \\ A. K. Tzioumis, ${ }^{3}$ R. H. Ferris, ${ }^{3}$ E. A. KING,${ }^{5}$ J. E. J. Lovell, ${ }^{5}$ P. M. MCCulloch, ${ }^{5}$ \\ K. J. Johnston, ${ }^{6}$ K. A. Kingham, ${ }^{6}$ J. O. Martin, ${ }^{6}$ G. L. White, \\ P. A. Jones, ${ }^{7}$ F. Arenou, ${ }^{4}$ M. Froeschlé, ${ }^{8}$ J. Kovalevsky, ${ }^{8}$ \\ C. MARTin, ${ }^{8}$ L. LindeGRen, ${ }^{9}$ AND S. SöDERHJELM ${ }^{9}$ \\ Received 1997 April 21; accepted 1997 July 14
}

\begin{abstract}
We report submilliarcsecond-precise astrometric measurements for the late-type star $\mathrm{AB}$ Doradus via a combination of VLBI (very long baseline interferometry) and HIPPARCOS data. Our astrometric analysis results in the precise determination of the kinematics of this star, which reveals an orbital motion readily explained as caused by gravitational interaction with a low-mass companion. From the portion of the reflex orbit covered by our data and using a revised mass of the primary star $\left(0.76 M_{\odot}\right)$ derived from our new value of the parallax ( 66.3 mas $<\pi<67.2$ mas), we find the dynamical mass of the newly discovered companion to be between 0.08 and $0.11 M_{\odot}$. If accurate photometric information can be obtained for the low-mass companion, our precise mass estimate could serve as an accurate calibration point for different theoretical evolutionary models of low-mass objects. This represents the first detection of a low-mass stellar companion using VLBI, a technique that will become an important tool in future searches for planets and brown dwarfs orbiting other stars.
\end{abstract}

Subject headings: astrometry — stars: individual (AB Doradus) — stars: kinematics stars: low-mass, brown dwarfs - techniques: interferometric

\section{INTRODUCTION}

The recent discoveries of several possible planetary or brown dwarf candidates using pulsar timing (Wolszczan \& Frail 1992) or optical spectroscopy (Mayor \& Queloz 1995; Marcy \& Butler 1996) have yielded a spectacular advance in the search for low-mass stellar companions. However, pulsar timing and optical spectroscopy do not yield unambiguous estimates of companion masses, because of the undetermined orbital inclination. Moreover, alternate explanations may exist for the radial velocity variations (Gray 1997). Companion masses can be uniquely determined by astrometric measurements of the motions of stars in the plane of the sky, which provide a full set of orbital elements. Submilliarcsecond VLBI (very long baseline interferometry) astrometry, using phase-referencing techniques (Lestrade et al. 1990), of weakly emitting radio stars has reached precisions better than 1 mas and should be a powerful tool for searching for very low mass objects.

Since 1992, we have been monitoring, with milliarcsecond precision, the radio positions of several Southern Hemisphere radio stars with VLBI (Guirado et al. 1996). The

\footnotetext{
${ }^{1}$ Jet Propulsion Laboratory, California Institute of Technology, 4800 Oak Grove Drive, Pasadena, CA 91109.

${ }^{2}$ Departament d'Astronomía i Astrofísica, Universitat de València, E-46100 Burjassot, Valencia, Spain.

${ }^{3}$ Australian Telescope National Facility, P.O. Box 76, Epping, NSW 2121, Australia.

${ }^{4}$ Observatoire de Paris, Section de Meudon, Place Jules Janssen, F-92195 Meudon Cedex, France.

${ }^{5}$ Department of Physics, University of Tasmania, GPO 252C, Hobart, Tasmania 7001, Australia.

${ }^{6}$ US Naval Observatory, 3450 Massachusetts Avenue, NW, Washington, DC 20392.

${ }^{7}$ University of Western Sydney Nepean, P.O. Box 10, Kingswood, NSW 2747, Australia.

${ }^{8}$ Observatoire de la Côte d'Azur, Avenue Copernic, F-06130 Grasse, France.

${ }^{9}$ Lund Observatory, Box 43, S-22100 Lund, Sweden.
}

main purpose of these astrometric observations is to contribute to the determination of the link between the celestial radio reference frame, defined by the VLBI positions of extragalactic radio sources, and the optical reference frame, defined by the positions of the stars observed by the European Space Agency's astrometric satellite HIPPARCOS (Lindegren \& Kovalevsky 1995; Lestrade et al. 1995). One of the stars of this program is AB Doradus (= HD $36705=$ HIP 25647), an active K0 star. Based on its high rotation rate $\left(P_{\text {rot }}=0.514\right.$ days, $\left.v \sin i \sim 100 \pm 5 \mathrm{~km} \mathrm{~s}^{-1}\right)$, lithium abundance (Rucinski 1985; Vilhu, Gustafsson, \& Edvardsson 1987), and membership in the Pleiades moving group (Innis et al. 1985), AB Dor is commonly accepted as a young pre-main-sequence star coeval with the stars of the Pleiades ( $70 \mathrm{Myr})$. AB Dor has an apparent physical companion, Rossiter 137B, a dM4e star that is also a rapid rotator $\left(P_{\text {rot }} \leq 0.38\right.$ days), separated by only $10^{\prime \prime}$ on the sky from AB Dor (Lim 1993). The two stars are believed to be associated based on their common proper motions and common radial velocities (Innis et al. 1985; Innis, Thompson, \& Coates 1986).

In this paper, we describe the results of our astrometric analysis, which reveals an acceleration in the position of $A B$ Dor that can be readily explained as caused by gravitational interaction with a low-mass companion.

\section{VLBI OBSERVATIONS AND DATA REDUCTION}

We performed VLBI measurements of AB Dor at 8.4 $\mathrm{GHz}$ at multiple epochs with an Australian array of radio telescopes (see Table 1). For each experiment, we interleaved observations of the strong background radio source PKS 0516-621 using, typically, a 250 s duty cycle that consisted of $140 \mathrm{~s}$ on AB Dor, $70 \mathrm{~s}$ on $0516-621$, and a $20 \mathrm{~s}$ slew time between each source. The data were correlated at the Mark IIIA correlator of the US Naval Observatory in Washington, DC. 
TABLE 1

Phase-Referenced Vlbi Observations of AB Doradus

\begin{tabular}{|c|c|c|c|c|}
\hline Epoch & Antennas $^{\mathrm{a}}$ & Recording Mode ${ }^{\mathrm{b}}$ & $\begin{array}{c}\text { Integration Time } \\
\text { (hr) }\end{array}$ & $\mathrm{SNR}^{\mathrm{d}}$ \\
\hline 1992 Apr 25 (1992.23) & $\mathrm{T}, \mathrm{H}, \mathrm{P}$ & MkIII A & 2.7 & 13 \\
\hline 1992 Sep $6(1992.68) \ldots \ldots \ldots$ & $\mathrm{T}, \mathrm{H}, \mathrm{P}$ & MkIII A & 2.9 & 25 \\
\hline 1993 Feb $14(1993.12) \ldots \ldots$ & $\mathrm{T}, \mathrm{H}$ & MkIII B & 2.7 & 10 \\
\hline 1994 Oct $24(1994.81) \ldots \ldots \ldots$ & $\mathrm{T}, \mathrm{H}, \mathrm{P}$ & MkIII B & 7.7 & 15 \\
\hline 1995 Feb $21(1995.14) \ldots \ldots$ & $\mathrm{T}, \mathrm{H}$ & MkIII B & 1.7 & 8 \\
\hline 1996 Feb 24 (1996.15) ...... & $\mathrm{T}, \mathrm{H}$ & MkIII A & 3.8 & 90 \\
\hline 1996 May $15(1996.37) \ldots \ldots$ & $\mathrm{T}, \mathrm{H}$ & MkIII A & 2.1 & 25 \\
\hline
\end{tabular}

${ }^{a}$ The symbols correspond to the following Australian antennas: (T) 70 m NASA Deep Space Network antenna at Tidbinbilla; (H) $26 \mathrm{~m}$ antenna of Mount Pleasant Observatory at Hobart; (P) 64 $\mathrm{m}$ ATNF antenna at Parkes.

${ }^{\mathrm{b}}$ We used the Mark III system (Rogers et al. 1983) to record 28 adjacent $2 \mathrm{MHz}$ channels covering 8402.99-8458.99 MHz (mode A) or 14 adjacent $2 \mathrm{MHz}$ channels covering $8402.99-8430.99 \mathrm{MHz}$ (mode B). Right-circular polarization (IEEE convention) was recorded.

c Total integration time resulting from the coherent addition of the VLBI data by using the phase-reference mapping technique.

d Signal-to-noise ratio of the AB Dor detections.

For the astrometric analysis, we followed the phasereferencing VLBI technique described by Lestrade et al. (1990) and used the software SPRINT, developed by J.-F. L. We outline briefly this analysis. First, we used the most accurate parameters to model the geometry of the interferometric array (reference source coordinates, antenna positions, and Earth orientation parameters were obtained from the International Earth Rotation Service; Feissel \& Essaïfi 1996), and the propagation media in order to produce theoretical estimates of the visibility phase that were subtracted from the observed estimates. Second, we interpolated the residual quasar phases (observed minus theoretical values) to the times of the star observations and subtracted them from the star fringe phases to form the differential residual fringe phase. Third, we carried out a Fourier transform of the resulting visibilities. This provided us with a phasereferenced map of the star whose coordinates are referred to the PKS 0516-621 position. The relative position of the star is then found by measuring the coordinates of the brightness peak on the phase-referenced map. Extensive error analysis at each observational epoch has been carried out to determine the uncertainties of the relative coordinates due to errors inherent in the propagation media, the reference source radio structure, and the geometry of the interferometry array. These uncertainties, ranging from 0.3 to 1.7 mas, were 5-7 times larger than the errors due to the signal-to-noise ratio of our VLBI data. The resulting positions and standard deviations are shown in Table 2.

\section{ORBIT DETERMINATION}

The measured VLBI coordinates of AB Dor (Table 2), referred to the IERS radio reference frame through the coordinates of PKS 0516-621, have been used to derive the star's proper motion, parallax, and position at a reference epoch (1993.00) via a weighted least-squares fit. In our first analysis, the root mean square (rms) of the postfit residuals was about 5 times greater than that of the other southern stars included in our program (Guirado et al. 1996), indicating that proper motion and parallax alone did not suffice to account for the trajectory of $A B$ Dor in the sky. The systematic signature, both in right ascension and declination, of the postfit residual positions of AB Dor led us to consider that the excursions were produced by the gravitational effect of a companion other than Rst 137B in orbit around AB Dor.

In order to enlarge our observational time span, we augmented the number of data points by using positions of $A B$ Dor measured by HIPPARCOS at several epochs over the mission's lifetime (1990.2-1993.3), during a time interval not covered by the VLBI data. In the final HIPPARCOS solu-

TABLE 2

Estimates OF THe Position of AB Doradus ${ }^{\mathrm{a}}$

\begin{tabular}{clcc}
\hline \hline Epoch & Technique & $\alpha(\mathrm{J} 2000)$ & $\delta(\mathrm{J} 2000)$ \\
\hline $1990.3888 \ldots \ldots$ & HIPPARCOS & $52844.77474 \pm 0.00026$ & $-652656.2416 \pm 0.0007$ \\
$1990.5640 \ldots \ldots$ & HIPPARCOS & $52844.78652 \pm 0.00025$ & $-652656.2272 \pm 0.0007$ \\
$1991.0490 \ldots \ldots$ & HIPPARCOS & $52844.77578 \pm 0.00024$ & $-652656.2615 \pm 0.0007$ \\
$1991.5330 \ldots \ldots$ & HIPPARCOS & $52844.78942 \pm 0.00025$ & $-652656.0757 \pm 0.0008$ \\
$1992.0180 \ldots \ldots$ & HIPPARCOS & $52844.78202 \pm 0.00024$ & $-652656.1160 \pm 0.0009$ \\
$1992.2329 \ldots \ldots$ & VLBI & $52844.77687 \pm 0.00019$ & $-65^{\circ} 26^{\prime} 56.0049 \pm 0.0007$ \\
$1992.6849 \ldots \ldots$ & VLBI & $52844.80124 \pm 0.00018$ & $-65^{\circ} 26^{\prime} 55.9395 \pm 0.0006$ \\
$1993.1233 \ldots \ldots$ & VLBI & $52844.78492 \pm 0.00024$ & $-65^{\circ} 26^{\prime} 55.9137 \pm 0.0008$ \\
$1994.8137 \ldots \ldots$ & VLBI & $52844.81768 \pm 0.00019$ & $-65^{\circ} 26^{\prime} 55.6866 \pm 0.0005$ \\
$1995.1425 \ldots \ldots$ & VLBI & $52844.80247 \pm 0.00027$ & $-65^{\circ} 26^{\prime} 55.6248 \pm 0.0011$ \\
$1996.1507 \ldots \ldots$ & VLBI & $52844.81137 \pm 0.00013$ & $-65^{\circ} 26^{\prime} 55.4852 \pm 0.0003$ \\
$1996.3607 \ldots \ldots$ & VLBI & $52844.81776 \pm 0.00018$ & $-65^{\circ} 26^{\prime} 55.3785 \pm 0.0010$ \\
\hline
\end{tabular}

NotE.-Units of right ascension are hours, minutes, and seconds, and units of declination are degrees, arcminutes, and arcseconds.

${ }^{a}$ VLBI positions of AB Dor were determined with reference to the IERS coordinates of the background radio source PKS 0516-621 ( $\left.\alpha=5^{\mathrm{h}} 16^{\mathrm{m}} 44^{\mathrm{s}} .926178, \delta=-62^{\circ} 7^{\prime} 5^{\prime \prime} .38930\right)$. 
TABLE 3

J2000.0 Astrometric ${ }^{\mathrm{a}}$ AND Orbital Parameters OF AB DORADUS

\begin{tabular}{|c|c|}
\hline Parameter & Value \\
\hline \multicolumn{2}{|l|}{ Astrometric: } \\
\hline$\alpha^{\mathrm{b}} \ldots \ldots \ldots$ & $52844.7948-44.7969$ \\
\hline$\delta^{\mathrm{b}}$ & $-652655.933-55.914$ \\
\hline$\mu_{x}\left(\mathrm{~s} \mathrm{yr}^{-1}\right) \ldots \ldots$ & $0.0074-0.0083$ \\
\hline$\mu_{\delta}\left(\operatorname{arcsec} \mathrm{yr}^{-1}\right) \ldots \ldots$ & $0.130-0.145$ \\
\hline$\pi(\operatorname{arcsec}) \ldots \ldots \ldots \ldots$ & $0.0663-0.0672$ \\
\hline \multicolumn{2}{|l|}{ Orbital: } \\
\hline$P(\mathrm{yr}) \ldots \ldots$ & $6.5-27.5$ \\
\hline$a_{1}(\operatorname{arcsec}) \ldots \ldots \ldots \ldots$ & $0.021-0.075$ \\
\hline 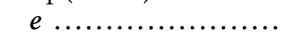 & $0.28-0.78$ \\
\hline$i(\operatorname{deg}) \ldots \ldots \ldots \ldots \ldots \ldots$ & $59-71$ \\
\hline$\omega(\mathrm{deg}) \ldots \ldots \ldots \ldots \ldots$ & $77-127$ \\
\hline$\Omega(\mathrm{deg}) \ldots \ldots \ldots \ldots \ldots$ & $127-142$ \\
\hline$T_{0} \ldots \ldots \ldots \ldots \ldots \ldots$ & $1991.5-1992.2$ \\
\hline$M_{c}\left(M_{\odot}\right)^{\mathrm{c}} \ldots \ldots \ldots \ldots$ & $0.08-0.11$ \\
\hline
\end{tabular}

NotE.-Units of right ascension are hours, minutes, and seconds, and units of declination are degrees, arcminutes, and arcseconds.

${ }^{\text {a }}$ Astrometric parameters correspond to the mass center of the system AB Dor/AB Dor C.

${ }^{\mathrm{b}}$ Reference epoch is 1993.00 .

$c$ Mass range obtained from the period and semimajor axis of each plausible orbit via Kepler's third law. The mass adopted for the central star, AB Dor, was $0.76 M_{\odot}$.

tion (ESA 1997), aligned with the IERS reference frame (Kovalevsky et al. 1997) as are the VLBI data, the motion of the photocenter of $\mathrm{AB}$ Dor was found to be nonlinear, corroborating the signature apparent in the VLBI residuals and showing that the star was an astrometric double. There were 55 individual data points for this star that were combined to obtain independent position estimates at five different epochs during the HIPPARCOS mission (see Table 2). The standard deviations of these estimates are about 1.5 mas in right ascension and 0.8 mas in declination. We note that the latter standard deviations also account for the uncertainty of the alignment between the radio and the optical reference frames.

To determine the orbital elements of the reflex motion of AB Dor, we combined both VLBI and HIPPARCOS data sets to estimate simultaneously the parameters that describe the system via a weighted least-squares analysis based on the Thiele-Innes method (Green 1985). These parameters include five astrometric parameters and seven orbital elements. The astrometric parameters are the two position coordinates, the two proper-motion components, and the parallax of the mass center of the system (central star and low-mass companion). The orbital elements are the period $(P)$, semimajor axis $\left[a_{1}=a M_{c}\left(M_{1}+M_{c}\right)^{-1}\right.$, with $a$ the semimajor axis of the relative orbit, $M_{1}$ the mass of AB Dor, and $M_{c}$ the mass of the low-mass companion], eccentricity $(e)$, inclination $(i)$, argument of the periastron $(\omega)$, position angle of the node $(\Omega)$, and epoch of periastron passage $\left(T_{0}\right)$. This simultaneous fit has the advantage that any sinusoidal behavior of the data is not absorbed by proper-motion and parallax effects (Black \& Scargle 1982). The result of this fit demonstrated that our joint VLBI/HIPPARCOS data set did not cover a full orbit, and that orbits with periods longer than 7 years fitted the data equally well.

To investigate the possible orbits for $\mathrm{AB}$ Dor consistent with the data, we used the Thiele-Innes method to treat the
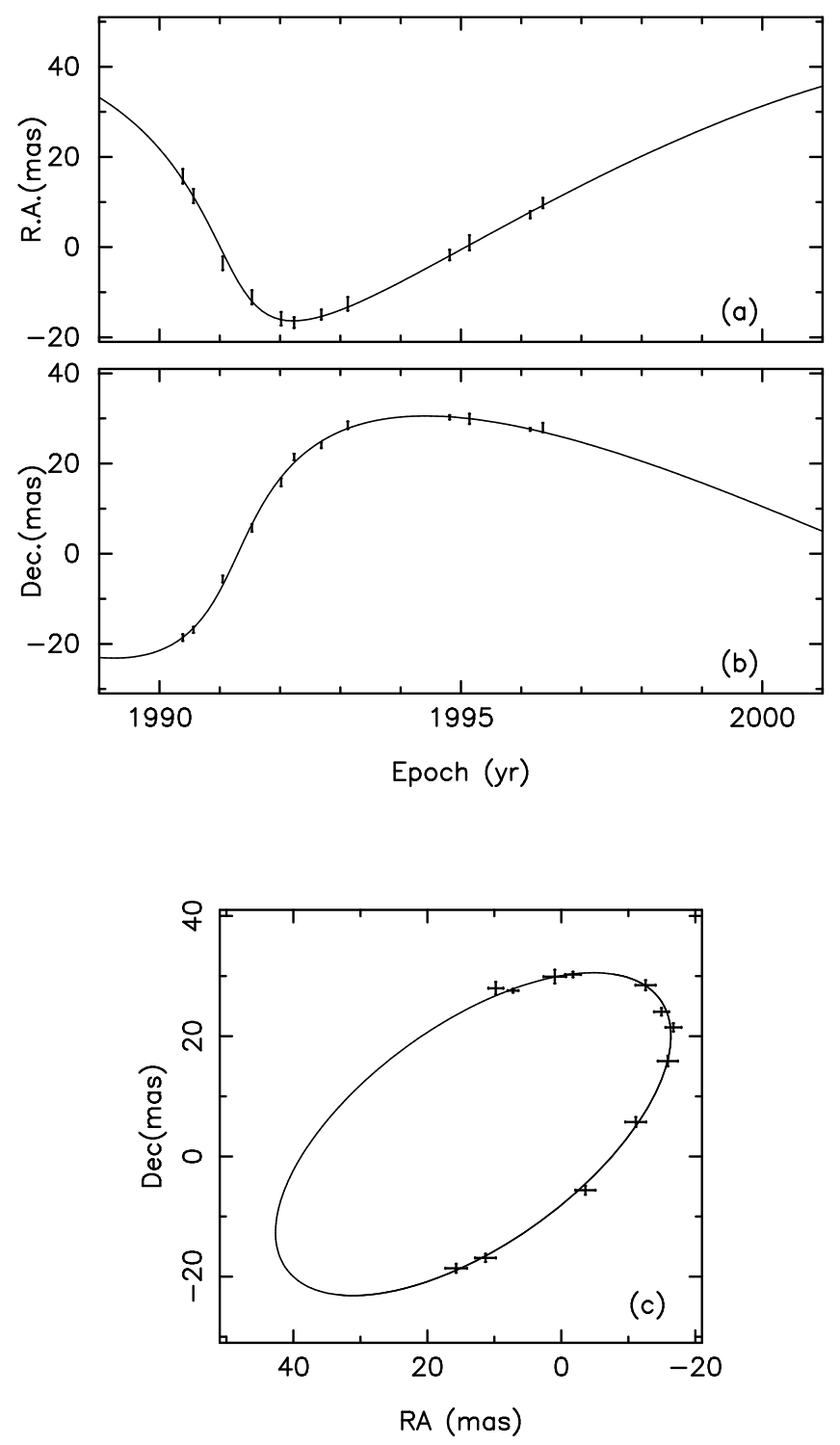

FIG. 1.-Orbit of the reflex motion of AB Dor corresponding to a companion mass of $0.094 M_{\odot} .(a, b)$ Right ascension and declination, respectively, of $\mathrm{AB}$ Dor for the observation epochs after subtracting proper-motion and parallax effects. The five earliest epochs correspond to HIPPARCOS data. The line corresponds to the least-squares fitted reflex orbital motion of AB Dor. (c) The apparent orbit, also with proper-motion and parallax effects subtracted.

nonlinear equations of the elliptic motion. This method has the advantage that it distinguishes between the three nonlinear orbital parameters $\left(P, e\right.$, and $\left.T_{0}\right)$ and the four linear Thiele-Innes coefficients that are the combinations of $a, i, \omega$, and $\Omega$. Consequently, the dimension of the parametric space to be searched is reduced from seven to three. Practically, we made multiple weighted least-squares fits; each fit had fixed values for the three nonlinear orbital elements while we solved for the four Thiele-Innes coefficients and the five astrometric parameters. We sampled a wide range of the parametric space: $6.5 \mathrm{yr}<P<27.5 \mathrm{yr}$ by steps of 5 days; $1990.0<T_{0}<1990.0+P$ by steps of 20 days; $0<e<1$ by steps of 0.005 days. We selected as plausible solutions those whose $\chi^{2}$ difference with the minimum $\chi^{2}$ was less than $15 \%$ of the latter value $(0.94$ mas). The resulting ranges of the astrometric parameters and orbital elements are given in 
Table 3. For each plausible orbit, we used Kepler's third law $\left[M_{c}^{3} /\left(M_{1}+M_{c}\right)^{2}=a_{1}^{3} / P^{2}\right]$ to estimate the mass $M_{c}$ of the unseen companion, AB Dor C. For this analysis, we used our new, more accurate, value of the parallax (see Table 3) to scale the mass of the central star AB Dor given by Vilhu et al. (1987) to $0.76 \pm 0.02 M_{\odot}$. We found that the mass of $\mathrm{AB}$ Dor $\mathrm{C}$ was constrained to the range $0.08-0.11 M_{\odot}$. This tight constraint on the mass for $\mathrm{AB}$ Dor $\mathrm{C}$ could not be obtained from either the VLBI or HIPPARCOS data sets alone. An orbit corresponding to a mass of $\mathrm{AB}$ Dor $\mathrm{C}$ near the center of the mass interval is displayed in Figure 1. We are continuing to obtain more VLBI data to narrow the range of the mass estimate.

We checked the sensitivity of our solution against a different choice of epochs of the HIPPARCOS positions by repeating the astrometric analysis with HIPPARCOS positions at epochs other than those shown in Table 2; the new results do not change significantly the ranges presented in Table 3. Regarding the astrometric parameters, the proper motion in right ascension and declination found in our analysis are consistent with long-term optical values (White et al. 1988) to within twice the standard deviation of the optical estimates. Also, our new parallax coincides with previously reported values at the limit of the quoted uncertainties (Innis et al. 1986). Finally, we note that our estimates given in Table 3 might be affected by a possible inconsistency between the two different data types, VLBI and HIPPARCOS. Since there is no overlapping between the portions of the orbit sampled by each data type, it is difficult to estimate the effect of such an inconsistency. However, this effect is unlikely to be significant, given the high precision of the alignment between the radio and optical reference frames (Kovalevsky et al. 1997).

\section{ALTERNATIVE EXPLANATIONS}

Some alternative explanations for the shifts in positions of AB Dor, such as stellar pulsation or surface activity, can be ruled out, because the smallest semimajor axis for the possible orbits investigated is $\sim 20$ mas, equivalent to about 60 stellar radii. The gravitational interaction of $A B$ Dor with Rst 137B can be also ruled out, as we calculate the wobble produced by Rst 137B on AB Dor to have an amplitude of $\sim 3^{\prime \prime}$ and a $\sim 2000 \mathrm{yr}$ period, given the common parallax and the mass for both stars (for this estimate, we scaled the mass of Rst 137B given by Vilhu et al. 1987 to $0.21 M_{\odot}$ ). Most of this orbital motion appears as a linear position drift during the short (compared with the $2000 \mathrm{yr}$ period) time span of our observations, and it is absorbed by the proper-motion estimate. The corresponding acceleration, about 0.03 mas $\mathrm{yr}^{-2}$, produces a shift of less than 0.5 mas in our 6 yr data span and, therefore, is not detectable in our present measurements. However, the gravitational interaction with Rst 137B will become significant after a few more years of observation, and we plan to include this effect in future astrometric analyses.

\section{DISCUSSION}

With a mass between 0.08 and $0.11 M_{\odot}, \mathrm{AB}$ Dor $\mathrm{C}$ is one of the few low-mass objects near the hydrogen-burning limit whose mass has been determined dynamically (Henry \& McCarthy 1993). AB Dor C offers a rare opportunity to combine a precise mass determination with photometric and/or spectral information for a young low-mass object. In particular, a suitable photometric calibration would locate this object in a mass-luminosity diagram and would add a new, precise point to the data given by Henry \& McCarthy (1993) for calibration of the low end of the main sequence (D’Antona \& Mazitelli 1985; Burrows et al. 1993; Baraffe et al. 1996).

An approximate expected magnitude for $\mathrm{AB}$ Dor $\mathrm{C}$ can be calculated from our mass estimate and assumed age ( $\sim 70 \mathrm{Myr}$, assuming that $\mathrm{AB}$ Dor and $\mathrm{AB}$ Dor $\mathrm{C}$ are coeval) by using theoretical evolutionary tracks for very low mass stars and brown dwarfs. Model X of Burrows et al. (1993) yields a luminosity for AB Dor C of $\log \left(L / L_{\odot}\right) \sim$ -2.5 and an effective temperature of $\sim 3000 \mathrm{~K}$. Taking the bolometric corrections derived for other very low mass stars in the Pleiades (Hamilton \& Stauffer 1993), and using our parallax determination, we can estimate the expected apparent magnitudes of $\mathrm{AB}$ Dor $\mathrm{C}$ to be $m_{V} \sim 16$ and $m_{K} \sim 9$. The magnitude difference between $\mathrm{AB}$ Dor $\left(m_{V}=\right.$ 6.95) and its dark companion, along with their separation, ranging from 0.2 to 0 ".7, makes the direct detection of $A B$ Dor $C$ unlikely with ground-based telescopes and very near the limits of the capabilities of the Hubble Space Telescope (HST) Wide Field Planetary Camera 2 (Schroeder \& Golimowski 1996). The contrast of the sources is more favorable for detection at near-infrared wavelengths $\left(m_{K}=6\right.$ and 9 for $\mathrm{AB}$ Dor and $\mathrm{AB}$ Dor $\mathrm{C}$, respectively), but the spatial resolution of ground-based Southern Hemisphere infrared devices is still too coarse. The Near-Infrared Camera and Multiobject Spectrometer, deployed on HST in 1997, appears to be the optimal method to obtain photometric information of AB Dor C.

We have shown that VLBI phase referencing, enhanced in this case with HIPPARCOS positions, is one of the most powerful techniques for searching for very low mass companions of orbiting stars. Significant improvement in the precision of this technique is potentially possible. Even with a similar precision to that achieved for AB Dor, the application of this technique to nearby $(<5 \mathrm{pc}) \mathrm{M}$ dwarfs could be extraordinary effective for detecting substellar companions with masses as low as 1 Jovian mass and orbital periods less than 5 years (Lestrade et al. 1996).

The research described herein was performed in part while J. C. G. held a National Research Council-NASA Resident Research Associateship award at the Jet Propulsion Laboratory, California Institute of Technology. Research at JPL is carried out under contract with the National Aeronautics and Space Administration. J. E. J. L. is supported by an Australian Postgraduate research award. The Australia Telescope is funded by the Commonwealth government for operation as a national facility managed by CSIRO. The optical contribution to this research is based on observations made with the European Space Agency HIPPARCOS satellite. 


\section{REFERENCES}

Baraffe, I., Chabrier, G., Allard, F., \& Hauschildt, P. H. 1996, ApJ, 446, L35

Black, D. C., \& Scargle, J. D. 1982, ApJ, 263, 854

Burrows, A., Hubbard, W. B., Saumon, D., \& Lunine, J. I. 1993, ApJ, 406, 158

D'Antona, F., \& Mazzitelli, I. 1985, ApJ, 296, 502

ESA. 1997, The Hipparcos and Tycho Catalogues (ESA SP-1200) (Noordwijk: ESA)

Feissel, M., \& Essaïfi, N., eds. 1996, IERS Annual Report for 1995 (Paris: Obs. Paris)

Gray, D. F. 1997, Nature, 385, 795

Green, R. M. 1985, Spherical Astronomy (Cambridge: Cambridge Univ. Press)

Guirado, J. C., et al. 1996, in ASP Conf. Ser. 6, Radio Emission from the Stars and the Sun, ed. A. R. Taylor \& J. M. Paredes (San Francisco: ASP), 134

Hamilton, D., \& Stauffer, J. R. 1993, AJ, 105, 1855

Henry, T. J., \& McCarthy, W. 1993, AJ, 106, 773

Innis, J. L., Coates, D. W., Thompson, K., \& Robinson, R. D. 1985, Proc. Astron. Soc. Australia, 6, 156
Innis, J. L., Thompson, K. \& Coates, D. W. 1986, MNRAS, 223, 183

Kovalevsky, J., et al. 1997, A\&A, 323, 620

Lestrade, J.-F., et al. 1995, A\&A, 304, 182

Lestrade, J.-F., Phillips, R. B., Jones, D. L., \& Preston, R. A. 1996, J. Geophys. Res. E, 101, 14837

Lestrade, J.-F., Rogers, A. E. E., Whitney, A. R., Niell, A. E., Phillips, R. B., \& Preston, R. A. 1990, AJ, 99, 1663

Lim, J. 1993, ApJ, 405, L33

Lindegren, L., \& Kovalevsky, J. 1995, A\&A, 304, 189

Marcy, G. W., \& Butler, R. P. 1996, ApJ, 464, L147

Mayor, M., \& Queloz, D. 1995, Nature, 378, 355

Rogers, A. E. E., et al. 1983, Science, 219, 51

Rucinski, S. M. 1985, MNRAS, 215, 591

Schroeder, D. J., \& Golimowski, D. A. 1996, PASP, 108, 510

Vilhu, O., Gustafsson, B., \& Edvardsson, B. 1987, ApJ, 320, 850

White, G. L., Jauncey, D. L., Batty, M. J., Peters, W. L., \& Gulkis, S. 1988, PASP, 100,825

Wolszczan, A., \& Frail, D. A. 1992, Nature, 355, 145 